IN S T I T U T O

$\mathrm{DE}$

M E D I C I N A

T R O P I C A L

$\mathrm{DE}$

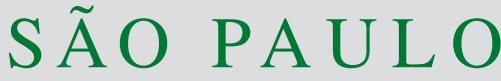

JOURNAL OF THE SÃO PAULO INSTITUTE OF TROPICAL MEDICINE

${ }^{1}$ Universidade Federal Fluminense, Niterói, Rio de Janeiro, Brazil

Correspondence to: Claudete Aparecida Araújo Cardoso Universidade Federal Fluminense, Rua Marquês de Paraná, 303, Centro, CEP 24.033-900, Niterói, RJ, Brazil Tel: +55 (21) 2629-9190

E-mail: claudetecardoso@id.uff.br

Received: 14 September 2021

Accepted: 2 December 2021

\section{Microcephaly caused by or associated with congenital infections in the last 20 years in Brazil: a systematic review}

\author{
Maria Dolores Salgado Quintans ${ }^{(1)}$, Arnaldo Costa Bueno ${ }^{\circledR 1}$, Claudete \\ Aparecida Araújo Cardoso ${ }^{1}$
}

\section{ABSTRACT}

This systematic review aimed to identify the pathogens causing or associated with congenital microcephaly in Brazil in the last 20 years due to the lack of official information by the Health Authorities and, as a consequence the uncertainty on the real infectious etiology of congenital microcephaly. A review protocol was prepared according to the PRISMA recommendation, using the PubMed, SciELO and LILACS databases to search for references presenting original data on microcephaly caused by or associated with congenital infectious in Brazil, using the descriptors "MICROCEPHALY AND INFECTION". The search ended on 30/Jun/2020. All selected titles were read in full and analyzed independently by the three reviewers. After searching the databases, 2,389 articles were selected for title review. Of these, 109 were excluded due to duplicates and 2,236 according to the criteria defined in the review. Only 44 met the eligibility criteria and were therefore read in full. Data extraction was performed on 10 articles, all published after 2015. Seven studies were literature reviews or case series, only two were case-control, and one was a cross-sectional study. As the studies focused on the period of the ZIKV epidemic in Brazil, the cases of congenital microcephaly between 2015 and 2017 were attributed to maternal infection by this virus when it was not possible to prove the presence of other etiological agents. Among the TORCH agents, a predominance of syphilis was observed. The analyzed studies did not add consistent information about the infectious causes or association of microcephaly in Brazil outside the period of ZIKV epidemic, revealing the need for more studies on the subject.

KEYWORDS: Microcephaly. Newborn. Congenital infection.

\section{INTRODUCTION}

The change in the etiological profile of congenital microcephaly due to infectious cause in Brazil during the ZIKA virus (ZIKV) epidemic brought light to this malformation that is very often associated with acute infectious conditions in pregnant women. The standardization of the measurement of the cephalic perimeter (CP) following changes established in the defining criteria of microcephaly at birth enabled an improvement on notifications of new cases by health professionals and a real estimate of cases of congenital microcephaly from $2015^{1}$. In Brazil, however, excepting for the period of National Emergency for ZIKV and microcephaly, there is a lack of publications regarding the identification of pathogens causing congenital microcephaly according to the etiological agent, and that can express the general and regional impact of ZIKV infections and other congenital infections in our country in recent decades. 
According to the World Health Organization (WHO), microcephaly is an anomaly in which the $\mathrm{CP}$ is less than two standard deviations (SD) than the reference for sex and gestation age ${ }^{1}$, and the severe form is defined as a $\mathrm{Z}$ score less than three $\mathrm{SD}^{2}$. The proposed percentile-based definition for microcephaly seems to be more accepted in Latin American countries and therefore, $\mathrm{CP}$ measured at birth below the third percentile in the reference curves and confirmed $24 \mathrm{~h}$ after birth may also be considered ${ }^{3}$. Microcephaly may be present at birth (congenital) or may develop after birth (acquired) ${ }^{4}$.

Microcephaly can also be classified according to its origin, into primary, when the brain does not grow to the expected size during pregnancy (before 32 weeks) and secondary, when the brain is the expected size at birth $^{5}$. Primary microcephaly is due to a decrease in the number of neurons generated during neurogenesis, while secondary microcephaly results from a reduction in the number of dendritic processes and synaptic connections. It is emphasized that tissue injury that occurs in early pregnancy, especially in the first trimester, due to infectious, toxic, or ischemic processes can disrupt subsequent brain development and result in macroscopic changes (malformation, disruption), and microscopic abnormalities (dysplasia) on the development of the central nervous system (CNS), often associated with microcephaly ${ }^{6}$. Congenital infections can lead to both, primary and secondary microcephaly, but this differentiation may not be possible if appropriate CP measures are not obtained ${ }^{2}$.

According to Ostrander et al. $^{7}$, congenital and perinatal infections represent the leading causes of permanent disability in children worldwide. Since the 1970s, the Centers for Disease Control and Prevention (CDC) created the acronym TORCH to indicate congenital infections caused by Toxoplasma, rubella virus, cytomegalovirus, and herpes simplex virus as potentially important etiologic agents involved in these conditions ${ }^{8}$. As early as 1974, Nahmias et al. ${ }^{9}$ presented estimates that, after excluding abortions and stillbirths, infection in newborns (NB) by TORCH occurred in 1 to $5 \%$ of births. At this time, the "O" in "TORCH" is also expanded to "Other" and includes hepatitis B virus (HBV), varicella zoster virus, syphilis, and later, the human immunodeficiency virus (HIV), among others. This is due to the recognition that prevention and treatment strategies can be provided to improve the health of the NB when these congenital infections are diagnosed early ${ }^{7-11}$. The acronym TORCH indicates what are the infectious agents that can produce similar clinical manifestations in affected NBs. Although the congenital rubella syndrome has almost disappeared in countries with universal immunization against this virus, TORCH agents, along with those recently recognized such as lymphocytic choriomeningitis virus and ZIKV, remain the leading causes of long-term neurodevelopmental impairment among infants worldwide ${ }^{7}$.

Several factors appear to influence malformations found in the neurological development of children affected by congenital infections. These include the tropism of the infectious agent, the immune status of the pregnant woman, and the timing of maternal infection (gestational age $)^{7}$. Placental infection and subsequent maternal-fetal barrier breakdown favor the invasion of the fetal circulation and dissemination of the pathogen that will reach various organs. The consequent triggered immune response can potentially lead to tissue damage. Leptomeningitis and brain necrosis can occur, causing lesions in the white matter, as well as ventriculomegaly and dystrophic calcifications. Loss of neurons results in microcephaly and the disruption of neuronal migration can lead to lissencephaly, polymicrogyria, schizencephaly and other cortical dysplasia ${ }^{7,12}$.

Regarding microcephaly, it is worth noting the attempts to standardize the clinical criteria involved in its description. The measurement of $\mathrm{CP}$ is based on the occipito-frontal measurement, with the head circumference always measured in centimeters avoiding rounding off the decimal points. Currently, WHO recommends adopting the criteria of the $21^{\text {st }}$ Intergrowth when gestational age is known or WHO Child Growth Standards when the gestational age cannot be estimated ${ }^{13}$. The definitions recently suggested by the Pan American Health Organization (PAHO) were presented for the purpose of public disclosure of the malformation and health surveillance, and they do not suggest implications for medical practice. It is also important to note that the normality limits of $\mathrm{CP}$ require standardized values for each gestational age; therefore, for full-term NB, the use of the WHO growth curves by sex is suggested. Specific reference curves by gestational age and sex (Fenton, 21st Intergrowth, etc.) should be used for preterm $\mathrm{NB}^{14}$. The difficulty in ascertaining the prevalence of microcephaly in the population is related to the inconsistency in the reported frequency, a lack of rigor in the measurement, as well as the incompleteness of clinical data that are contained in the reports generated by systematic data collection in the population. These are probably the most important factors limiting the comparability of microcephaly prevalence estimates, accounting for variability in observed rates around the world ${ }^{2}$.

In October 2015, the Brazilian government initiated joint epidemiological investigation actions in Pernambuco State, after observing the increasing number of microcephaly cases in that State. The first question was whether the 
observed increase could be casual, attributed to a simple fluctuation of numbers that simulated an epidemic, by simple coincidence. However, a first consultation to the Live Births Information System (SINASC), maintained by the Ministry of Health (MS), which receives birth data from live births declarations, confirmed that the numbers observed in October 2015 in Pernambuco, and they were much higher than the usual records ${ }^{15}$. On November 28, 2015, the MS recognized the relationship between the increased incidence of microcephaly in Brazil and ZIKV infection during pregnancy and on November 29 of the same year, MS changed the classification of this event, under the International Health Regulations (IHR), to a potential Public Health Emergency of International Importance (PISS). The resulting Protocol for Surveillance and Response to the Occurrence of Microcephaly and/or CNS Alterations incorporated the classification of reported cases according to the results of specific imaging and laboratory tests, discriminating them as confirmed cases of NB with microcephaly suggestive of being related to congenital infection (TORCH) or ZIKV. However, it was possible to classify cases as suspected ZIKV infections when the mother presented exanthema during pregnancy, and the NB presented signs compatible with congenital infection by any imaging method or laboratory tests, with non-reagent TORCH tests in samples from the NB and/or mother ${ }^{1}$. In these NBs, the specific laboratory diagnosis of ZIKV was mainly based on the detection of viral RNA from clinical specimens such as blood, CSF, urine and placenta. From another perspective, in imaging examinations, the main abnormalities suggestive of congenital infection can be observed both in ultrasound exams during pregnancy or transfontanellar ultrasounds, as well as in computed tomography (CT) or magnetic resonance imaging (MRI) of the skull. MRI shows changes that are not seen in skull $\mathrm{CT}$, allowing a better characterization of cortical changes and a better assessment of the white matter ${ }^{1}$. All the criteria described above were necessary for the notification of suspected cases during the ZIKV epidemic, when it was not possible to confirm all acute infections by specific laboratory tests in pregnant women and NBs.

At that time, an online Public Health Event Registration Form (RESP - Microcephalia) was made available and it remains in use until now, accessible only by the cases' Federal Unit (FU) of residence, so that all suspected cases of microcephaly related to ZIKV have been registered. This tool aims to allocate the notifications in a single place in order to allow proper management of information related to the event, and it is important to emphasize that this database, with respective notifications, is not an information system and does not replace the diagnostic investigation. The registration in the RESP enables Health Care providers to identify and monitor cases of microcephaly presenting with signs of neuropsychomotor developmental delay (NPMD), regardless of the cause of microcephaly ${ }^{1}$.

Nunes et al. ${ }^{15}$ reproduced data from the MS epidemiological report on February 12, 2016, with a cumulative total (in the years 2015 - 2016) of 5,079 reported cases of microcephaly and/or other CNS disorders, including cases possibly related to ZIKV and other infections. The Northeast region concentrated $98 \%$ of the municipalities with confirmed cases at that time. The most affected States in absolute numbers in descending order were Pernambuco, followed by Bahia, Rio Grande do Norte, Paraiba, Piaui, Alagoas, Ceara, Espirito Santo, Rio de Janeiro, Para, Goias, Mato Grosso do Sul and Rio Grande do Sul.

On May 11, 2017, the MS declared the end of the National Emergency for ZIKV and microcephaly ${ }^{16}$. By April 15 of that year, 7,911 cases of ZIKV had been reported nationwide, a $95.3 \%$ reduction from 2016, when there were 170,535 notifications. Microcephaly data showed an important reduction in the number of new cases reported every week since May 2016. At the peak of microcephaly cases in December 2015, a 135\% increase in notifications was recorded ${ }^{17}$.

Identifying the pathogens causing congenital microcephaly of infectious etiology that can cause permanent sequelae that are often preventable, can favor decision making and the adoption of regional public health measures for prevention. This systematic review of the literature aims to verify the pathogens associated with or causing congenital microcephaly of infectious etiology specifically in Brazil, in the last 20 years.

\section{MATERIALS AND METHODS}

A review protocol was prepared according to the PRISMA recommendation, registered in the PROSPERO platform under the number 192035. PubMed, SciELO, and LILACS platforms were searched on November 8, 2019 aiming to gathering bibliographic references presenting original data on the frequency of congenital microcephaly of infectious cause in Brazil, using the descriptors "MICROCEPHALY AND INFECTION" (Figure 1). Articles in Portuguese, English, Spanish and French were considered, and a filter of newborn and the infant's age was used for the population. No article type or publication date filters were used. The use of descriptors that amplified the search had the intention of choosing all articles considered relevant to answer the research question. Likewise, attention was paid to updating the search, with the last search being performed on June 30, 2020. 


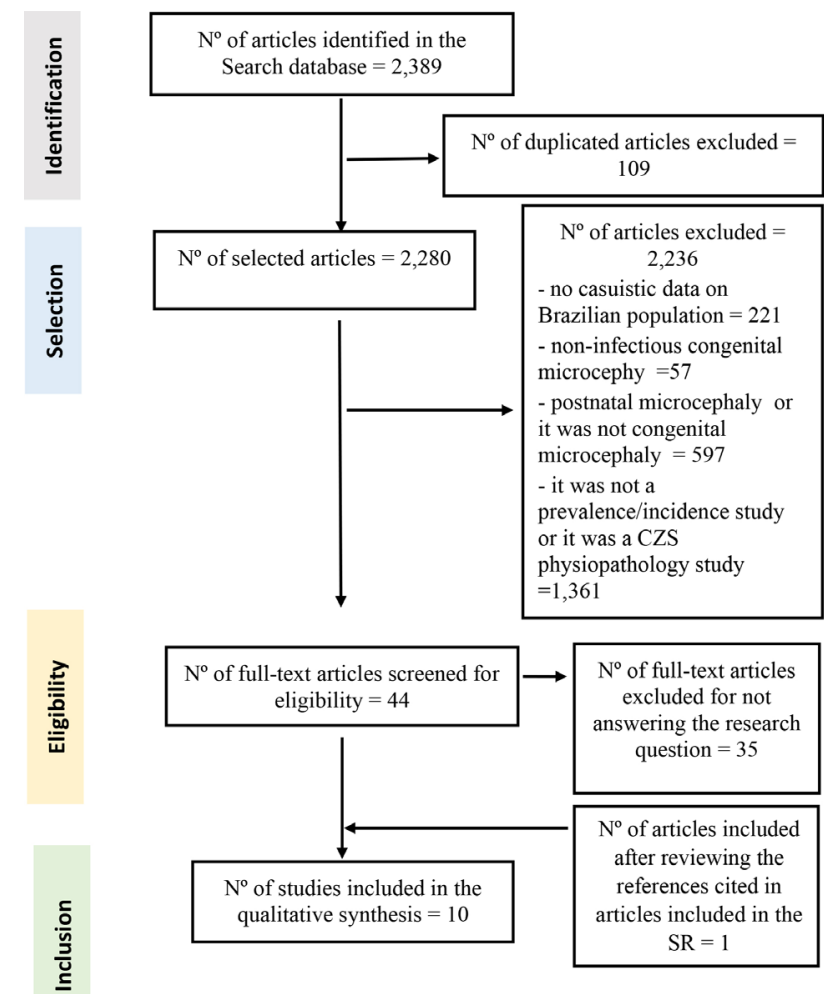

Figure 1 - Search strategy in PubMed, SciELO e LILACS data bases. $\mathrm{CZS}=$ congenital Zika syndrome; $\mathrm{SR}=$ systematic review.

Two reviewers independently analyzed the selected titles to determine those that should be included in the abstracts review. Disagreements were resolved by a third reviewer. The same protocol was followed for the selection of abstracts and articles for the full reading step. The selected articles were entirely read by the three reviewers, and then submitted to discussion regarding source, location and design of the study, objectives, population included, results found, limitations and quality of evidence by GRADE (Grading of Recommendations Assessment, Development and Evaluation). Only primary data from studies conducted in NBs diagnosed with microcephaly at birth resulting from a maternal congenital infection during pregnancy in the Brazilian population were included for final extraction of results. Only studies of prevalence/incidence/association of infectious microcephaly were included. Articles cited in the read texts and meeting these criteria were also included in the review. The data were extracted jointly by the three reviewers and were organized in an Excel table.

All excluded articles were categorized according to the following exclusion grounds: the article does not deal with a Brazilian population; the cases of microcephaly were not attributable to an infectious cause; the article did not mention the presence of NB with microcephaly, or microcephaly occurred in the postnatal period; and finally, it referred to the pathophysiology of Congenital Zika Syndrome (CZS) based on the causal relationship of ZIKV infection and microcephaly, as well as on the manifestations of CZS without specifying microcephaly in the population studied. After reading in full, case reports, protocols published by government agencies, epidemiological bulletins and articles with non-primary research data were also excluded.

The Kappa score $(\kappa)$ was calculated with near perfect agreement between the two reviewers in both the title and abstract exclusion phases [ $\kappa$ of 0.92 (95\% CI, 0.82-0.97) $]^{18}$.

The data extraction table was structured with the following fields:

- Author and year of publication;

- Study site: given that our question was restricted to cases occurring in Brazil, the study site refers to the location in which the cases of congenital microcephaly of infectious cause occurred;

- Study design: were categorized into systematic review with/without meta-analysis, literature review, cohort studies, case-control studies, cross-sectional studies and case series;

- Study objective: in which the objective selected by the study authors was achieved;

- Study period: the beginning and end dates of data collection by the authors were recorded by day, month, and year or only month and year, according to what was reported in the article;

- Study population;

- Total cases of congenital microcephaly in absolute numbers: in studies in which CNS malformations were analyzed, cases of congenital microcephaly were frequently not quantified and the information was not computed in the spreadsheet (data not reported);

- Total cases of microcephaly of infectious etiology in absolute numbers and percentages: in this field, both cases of microcephaly due to confirmed infectious origin and cases confirmed by epidemiological relationships during the ZIKV epidemic in Brazil were considered;

- Total cases of microcephaly due to infectious etiology with laboratory or radiological confirmation in absolute numbers and percentages: the objective of this field was to identify the infectious causes of microcephaly in cases in which it was possible to confirm the etiological agent of the infection in the mother or NB;

- Limitations: those described in the study text by the author of the reviewed article and/or limitations that are specific to our research question;

- GRADE: the evaluations related to the quality of evidence for the research question classified the studies as high, moderate, low and very low, according to the proposed criteria. The study design, the use of 
data obtained by indirect evidence, and the presence of measurement, recall, and outcome biases were considered.

The review presented here did not use statistical tools other than a simple percentage obtained in the EXCEL program where the results were tabulated.

\section{RESULTS}

After searching the databases, 2,389 articles were selected for title review (Figure 1). The titles selected in all three platforms were checked in order to eliminate duplicates, which resulted in 109 articles. Of the remaining 2,280 articles, 2,236 were excluded according to the criteria defined in the review. Only 44 met the eligibility criteria and were therefore read in full. Data extraction was performed on 10 articles.

The data extracted from the articles selected for the qualitative synthesis are presented in Table 1.

\section{DISCUSSION}

Several articles were published concomitantly or after the period of Public Health Emergency of ZIKV and microcephaly in Brazil ${ }^{2,7,12}$. The emergence of a new pathogen transmitted vertically to the fetus and directly associated with the occurrence of microcephaly in NBs in an extremely susceptible population has leveraged the number of published studies on the subject. According to this literature review, until today, it has not been possible to identify systematic review studies capable of determining the association or the causality between each transmitted infectious agent and congenital microcephaly in Brazil, consistent with its population profile and over the years. Although numerous, almost all publications in databases were unable to answer the target question of this review. For the primary endpoint of congenital microcephaly due to an infectious cause, it was possible to extract data in only 10 articles $^{19-28}$. All selected studies were published after the year 2015, with data obtained in the period between years 2015 and 2017, during the ZIKV epidemic in the country.

In the Morbidity and Mortality Weekly Report, a CDC report published in March 2016, Oliveira et al. ${ }^{20}$ reported that the prevalence of microcephaly at birth in Brazil had sharply increased during the years 2015-2016. The largest increment was seen in the Northeast region of the country, where the first reports of vertical transmission due to ZIKV occurred. In this publication, 574 cases of microcephaly detected by the surveillance system were analyzed, with temporal and geospatial evidence of a relationship between the presence of an exanthematous febrile illness caused by ZIKV during the first trimester of pregnancy, and an increased prevalence of microcephaly at birth. The prevalence of microcephaly in 15 States of the federation in which ZIKV transmission could be confirmed significantly exceeded that found in four States where it could not be confirmed (2.8 cases per 10,000 live births/ lives x 0.6 per 10,000). Magalhães et al. ${ }^{19}$ also used the official notifications of the MS to register the number of new cases of microcephaly, during the same period investigated in the present study in Brazil. The objective was to guide discussions on the evolution of both outbreaks in various regions of the country, based on the epidemiological bulletins published until then. Between November 2015 and July 2016, 8,301 cases of microcephaly and/or other neurological abnormalities were reported, with investigation completed in 5,171 (62.3\%) cases, of which 1,656 (32.0\%) were confirmed and 3,515 (68\%) were excluded. The etiology of these abnormalities at birth was confirmed in 255 (15.4\%) of cases in which ZIKV infection was confirmed by RT-PCR or positive serology in samples from the mother or the NB. At the end of the study, more than $1 / 3$ of the reported cases were still under investigation (37.7\%). Furthermore, it was not possible to exclusively isolate microcephaly cases from the other malformations found in $\mathrm{CZS}^{20}$. These two literature reviews and population-based studies evaluated cases of microcephaly reported nationwide.

In eight other studies, the origin of registered cases was distributed among States in the Northeast $(\mathrm{n}=6)$, Southeast $(\mathrm{n}=1)$ and South $(=1)$. Most studies $(80 \%)$ were literature reviews or case series, only two were case-control, and one was a cross-sectional study. In this context, we emphasize that we are probably facing duplicity of tabulated data, with a great chance of results published by more than one author being the same. Since the period of occurrence of microcephaly cases coincides, the reported serial cases are potentially the same as those of population-based studies. In fact, case series studies were fundamental to the registration of the first microcephaly cases caused by ZIKV in the Northeast region of Brazil, and to properly establish the causal link between the two. One of the case series initially published aimed to describe the first events of microcephaly possibly related to ZIKV in Recife, as well as the epidemiological profile of those mothers ${ }^{21}$. In 2015, from August $1^{\text {st }}$ to October $31^{\text {st }}, 40$ microcephaly cases were confirmed in the metropolitan region of Recife. From the total of 16 diagnostic tests performed for etiological definition, three were positive and one was inconclusive for syphilis, one had positive IgM serology for herpes ( 1 positive test in 10 tests performed), one had positive IgM serology for CMV (1 positive test in 19 tests performed), in addition to three inconclusive results by polymerase chain 


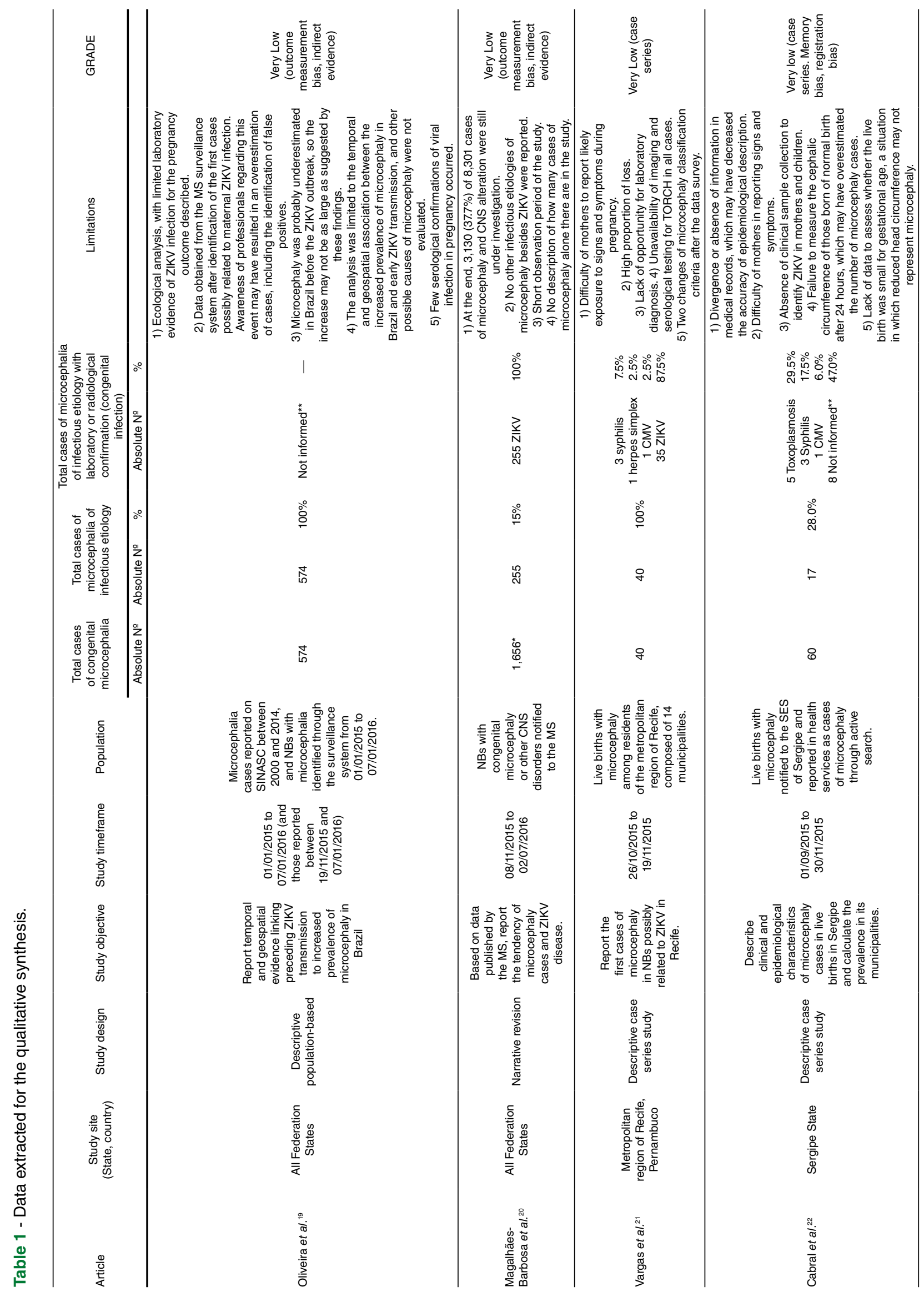




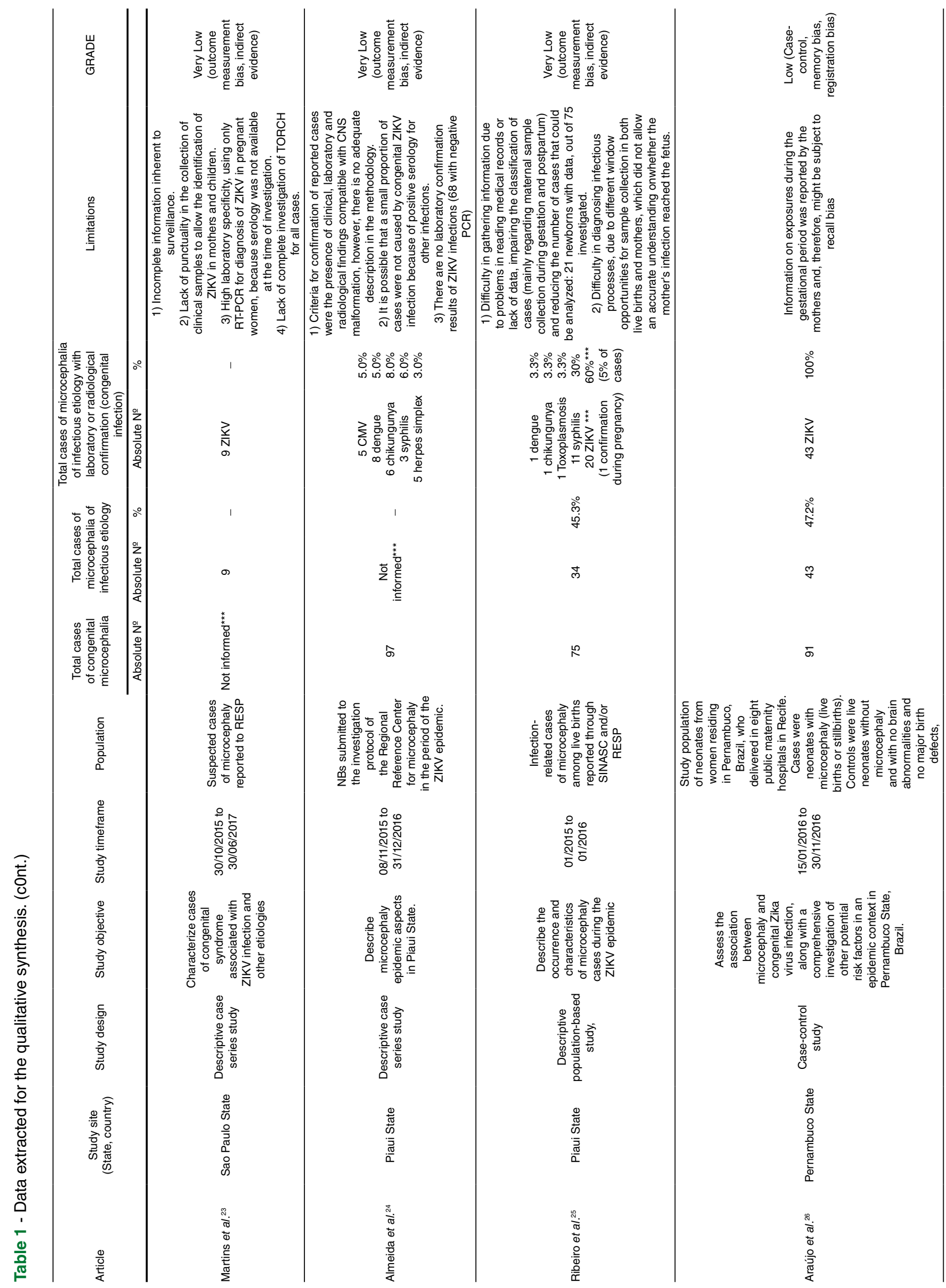




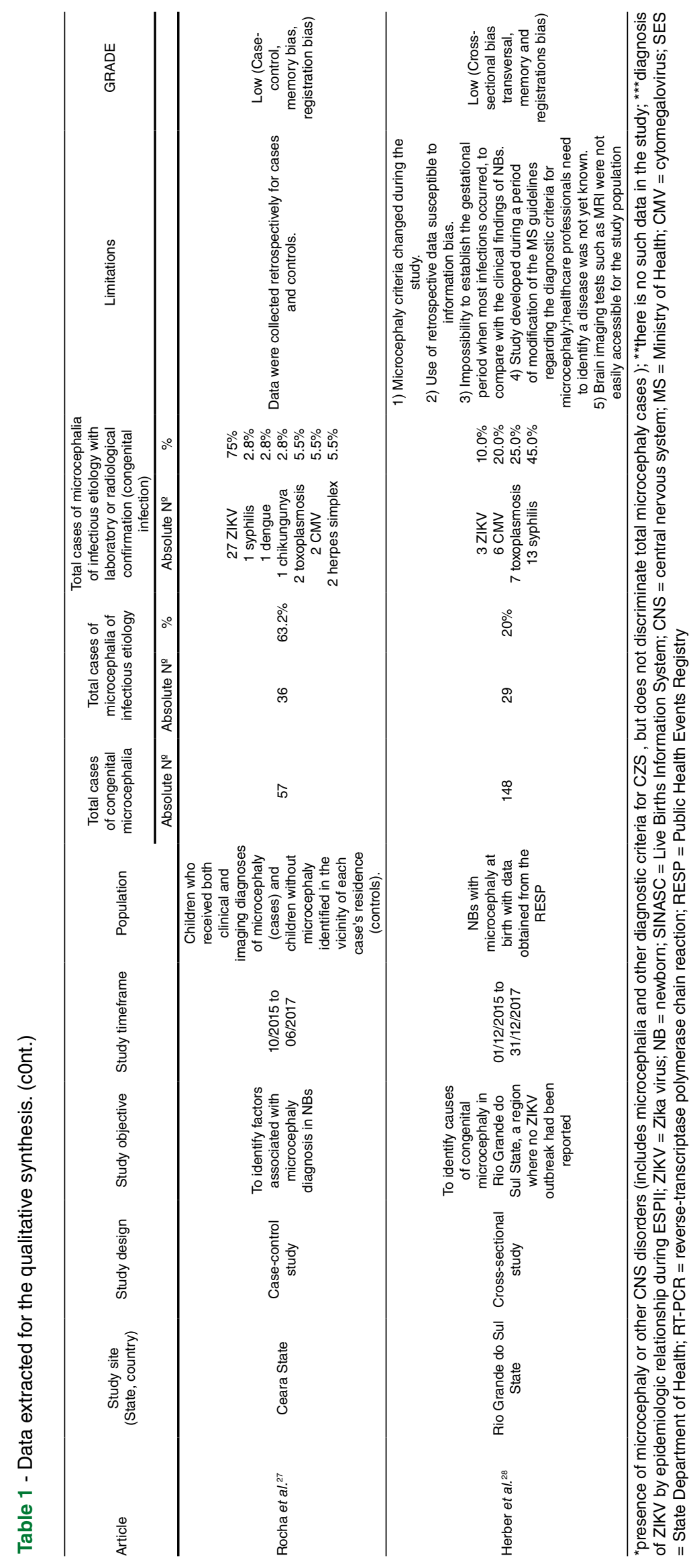


reaction (PCR) for CMV. The other TORCH agents tested were negative. Therefore, a total of 35 cases were associated to ZIKV infections, considering the outbreak of the disease in that region during the period studied. Mothers' difficulty to report the presence of signs and symptoms during pregnancy, the high proportion of evasion in the follow-ups, but mainly the lack of opportunity to perform a laboratory diagnosis in addition to the unavailability of imaging exams and serological tests for TORCH, were limiting factors to clarify the etiology of infections in all cases ${ }^{21}$. A study with a similar design was conducted concomitantly in Sergipe State, and the same operational problems for the etiological confirmation were found in the evaluation of 60 cases of microcephaly reported to that Health Secretariat ${ }^{22}$.

An article that should be highlighted is the crosssectional study by Herber et al. ${ }^{28}$ that aimed to identify the causes of congenital microcephaly during the period of Public Health Emergency in Brazil, in Rio Grande do Sul State, where no ZIKV outbreak was detected. During the study period, $15 \%$ of microcephaly cases $(n=39)$ with confirmed etiological infectious diagnosis and a predominance of congenital syphilis cases, with only three (1\%) confirmed cases of maternal infection by ZIKV.

Most studies $(n=8)$ used data obtained retrospectively, after the identification of microcephaly, through research in official forms (RESP) or through review of medical records and maternal interviews. Therefore, there may be low reliability in the medical records measured, as well as in the collection of information from mothers of NBs exposed to ZIKV through interviews that occurred several months after the episode of exanthema in pregnancy. During the period of public health emergency of international importance, there was a modification in the criteria for clinical diagnosis of microcephaly, which may have initially led to an overestimation of cases; however, it was not possible to observe any correction in the initial estimate, or any discarding of cases initially reported during the studies. The lack of accuracy was evident, both in the measurement of cases and in the diagnosis due to difficulties of clinical standardization for confirmation of microcephaly cases and the bias of memory of the interviewees.

Another important point for discussion is related to the fact that there was no description by most authors about the criteria used for confirmation of the etiology of microcephaly cases. For congenital infections confirmed by TORCH, the laboratory diagnostic methods described are those classically recommended in the literature based on the direct identification of the microorganism or on serology. Low compliance with prenatal care, lack of access to serology, and the long time elapsed between clinical diagnosis and laboratory testing may have limited the confirmation of congenital infections in these NBs. This finding is of great relevance when considering that most cases of diagnosed congenital microcephaly caused by ZIKV during the epidemic in Brazil were established through epidemiological relationships, and infection by infectious agents other than ZIKV was not irrefutable. The final report of a case-control study published by Araújo et $a l .{ }^{26}$ confirmed the association between microcephaly and congenital Zika virus infection. They provided evidence on the absence of an effect of other potential factors, such as exposure to pyriproxyfen or vacines. In addition, the casecontrol studies by Rocha $e t$ al. ${ }^{27}$ and the cross-sectional study by Herber et al. ${ }^{28}$ confirmed microcephaly due to ZIKV by laboratory and/or radiological methods.

Among the agents belonging to the TORCH group, a predominance in the diagnosis of congenital syphilis associated with congenital microcephaly was observed, specifically in the studies by Ribeiro et al. ${ }^{25}$ and Herber et $a l .{ }^{28}$. This information confirms the data from the latest Epidemiological Bulletin of Syphilis of the Ministry of Health (2020) that in Brazil, in general, in the last 10 years and especially from 2010, there has been a progressive increase in the incidence rate of congenital syphilis: in 2008, the rate was 2.0 cases/1,000 live births, and in 2018, they were more than four times higher, reaching 9.0 cases $/ 1,000$ live births, decreasing to 8.2 cases per 1,000 live births in 2019. The increased surveillance of syphilis cases in pregnant women and NBs is likely responsible for this phenomenon ${ }^{29}$. Although microcephaly is not among the frequent clinical manifestations of early congenital syphilis, coinfection should always be ruled out. In the case-control study by Araújo $\mathrm{et} \mathrm{al.} .^{26}$, no neonate tested positive for IgM anti-CMV, Toxoplasma gondii or rubella virus.

In the MS Epidemiological Bulletin of July 2020, during epidemiological weeks 45 of 2015, and 25 of 2020 (June 24, 2020), 19,072 suspected cases of congenital Zika syndrome (CZS) and other infectious etiologies were reported to the MS, of which 3,535 (18.5\%) were confirmed ${ }^{30}$. Thus, the possibility of congenital microcephaly cases caused by ZIKV is still present in our environment.

Another pertinent aspect is the possibility of diagnosing cases of microcephaly associated with ZIKV according to the presence of some typical lesions evidenced in image scans of the CNS. In their case series, Almeida et al. ${ }^{24}$ described lesions identified in skull CT of 95 children with confirmed diagnosis of microcephaly in comparison with the findings in 21 discarded cases of the disease. CT revealed intracranial calcifications in $78(82.1 \%)$ of 95 confirmed cases of microcephaly and in $1(4.8 \%)$ of 25 discarded cases $(\mathrm{p}<0.001)$. Transfontanellar US detected $6(30 \%)$ of the children with calcifications ${ }^{24}$. In the case- 
control study by Araújo et $a l^{26}{ }^{26}$ for the cases, the brain image was obtained by $\mathrm{CT}$ and was classified according to the presence or absence of major brain damage identified by physicians specialized in imaging diagnosis. The abnormalities included were calcification, ventriculomegaly, cortical malformation development (such as lissencephaly and polymicrogyria), and supposed vascular abnormalities. Controls were investigated by transfontanellar US. For 79 NBs with microcephaly, 21 (27\%) had major cerebral anomalies on CT. In their results, for $79 \mathrm{NBs}$ with microcephaly and imaging studies, 21 (27\%) had major cerebral anomalies on CT. Ten (43\%) of 23 cases that were positive for Zika virus had major cerebral abnormalities on CT, compared with $11(20 \%)$ of the 56 cases that tested negative for Zika virus ( $\mathrm{p}=029 ; \chi^{2}$ test comparing the frequency of abnormalities between NBs who were positive and negative for Zika virus). For the $\mathrm{MS}^{31}$, microcephaly cases suggestive of being related to congenital infections can be confirmed in the NBs, when abnormalities indicative of congenital infection are evident by any imaging method, in the absence of confirmatory laboratory results. In fetuses, the presence of brain calcifications and/or the presence of ventricular dilation and/or at least two of the following signs of posterior fossa abnormalities: cerebellar hypoplasia, cerebellar vermis hypoplasia, posterior fossa enlargement greater than $10 \mathrm{~mm}$, and agenesis/hypoplasia of corpus callosum on ultrasound examination are sufficient for case confirmation based on the radiological criterion of CNS infection by ZIKV. Many studies have based their estimates of confirmed cases of microcephaly during the ZIKV epidemic in Brazil on clinical-radiological criteria, without discriminating or excluding the criteria used.

Despite an evident decrease in the number of microcephaly cases reported after the epidemic, two cases associated with ZIKV were still confirmed in 2020. Regardless that the emergency period has ended, new cases of CZS continue to occur in the country, which justifies the continuity of surveillance, confirmation of infection, and publication of research on the subject ${ }^{30}$. It is essential to recognize the role of each infectious agent as a determinant of congenital microcephaly in our environment due to the potential risk of coinfection.

Restructuring the health system to offer care services to children with microcephaly is essential to ensure comprehensive care for their specific needs. Congenital malformations, including microcephaly, have always been notified through the Declaration of Live Births and these data are consolidated in the Information System on Live Births (SINASC) of the Ministry of Health. Given the need for suspected cases of microcephaly to be immediately notified to the Ministry of Health in the fight against ESPIN/
Zika, as of 2015 an online form called RESP-Microcephaly was made available by DATASUS, which was used by all State health departments in the context of the epidemic. Despite the mandatory notification of microcephaly at birth, the information regarding the investigation of cases and, consequently, regarding the causes associated with this malformation do not follow the same parity. Is there a lack of research, of adequate tools to confirm the etiology or it is just the unavailability of the data produced? It would be essential that the health departments, when ending the investigation of suspected or confirmed cases of microcephaly associated or not with preventable congenital infections, register these results on online platforms or in periodic epidemiological bulletins, enabling the appropriate adjustments of public policies aimed at this population.

\section{CONCLUSION}

The large number of studies during the ZIKV epidemic period does not measure the real association of congenital infections responsible for microcephaly at birth in our population over the last years. There is no official information publicly available about the conclusion of cases investigated by the Health Secretariats, so that the real infectious etiology of congenital microcephaly is unknown, except during the period of the Zika virus epidemic in the country. In our systematic literature review, it was also not possible to identify, in Brazil, consistent studies capable of translating the infectious causes of congenital microcephaly, which demonstrates an explicit lack of studies in this area. We emphasize the importance of more robust studies identifying different etiology of microcephaly of infectious causes in Brazil to plan public health policies according to the demand of our population.

\section{AUTHORS' CONTRIBUTIONS}

MDSQ, ACB and CAAC equally contributed to the manuscript draft. All authors were responsible for the bibliographic survey, review of the articles and data extraction.

\section{CONFLICT OF INTERESTS}

The authors declare that they have not conflict of interest to disclose. All authors reviewed, revised and provided final approval of the manuscript.

\section{FUNDING}

We declare no financial support regarding the publication 
of this paper, and the research did not receive any specific grant from funding agencies in the public, commercial or not-for-profit sectors.

\section{REFERENCES}

1. Brasil. Ministério da Saúde. Secretaria de Vigilância em Saúde. Departamento de Vigilância das Doenças Transmissíveis. Protocolo de vigilância e resposta à ocorrência de microcefalia relacionada à infecção pelo vírus Zika. Brasília: Ministério da Saúde; 2015. [cited 2021 Dec 2]. Available from: https:// portalarquivos2.saude.gov.br/images/pdf/2015/dezembro/09/ Microcefalia---Protocolo-de-vigil--ncia-e-resposta---vers--o1----09dez2015-8h.pdf

2. Devakumar D, Bamford A, Ferreira MU, Broad J, Rosch RE, Groce N, et al. Infectious causes of microcephaly: epidemiology, pathogenesis, diagnosis, and management. Lancet Infect Dis. 2018;18:e1-13.

3. Brasil. Ministério da Saúde. Secretaria de Vigilância em Saúde. Vírus Zika no Brasil: a resposta do SUS. Brasília: Ministério da Saúde; 2017. [cited 2021 Dec 2]. Available from: https://bvsms. saude.gov.br/bvs/publicacoes/virus_zika_brasil_resposta_sus. pdf

4. World Health Organization. Microcephaly. [cited 2021 Dec 2]. Available from: https://www.who.int/en/news-room/factsheets/detail/microcephaly

5. Woods CG. Human microcephaly. Curr Opin Neurobiol. 2004; $14: 112-7$

6. Passemard S, Kaindl AM, Verloes A. Microcephaly. Handb Clin Neurol. 2013;111:129-41.

7. Ostrander B, Bale JF. Congenital and perinatal infections. Handb Clin Neurol. 2019;162:133-53.

8. Nahmias AJ, Walls KW, Stewart JA, Herrmann KL, Flynt Jr WJ. The ToRCH complex-perinatal infections associated with toxoplasma and rubella, cytomegol- and herpes simplex viruses. Pediatr Res. 1971;5:405-6.

9. Nahmias AJ. The TORCH Complex. Hosp Pract. 1974;9:65-72.

10. Neu N, Duchon J, Zachariah P. TORCH infections. Clin Perinatol. 2015;42:77-103.

11. Bale JF Jr. Fetal infections and brain development. Clin Perinatol. 2009;36:639-53.

12. Mlakar J, Korva M, Tul N, Popović M, Poljšak-Prijatelj M, Mraz $\mathrm{J}$, et al. Zika virus associated with microcephaly. N Engl J Med. 2016;374:951-8

13. World Health Organization. Child growth standards. [cited 2021 Dec 2]. Available from: http://www.who.int/childgrowth/ standards/en/

14. World Health Organization. Avaliação de bebés com microcefalia no contexto do vírus Zika: orientações provisórias, 4 de março de 2016. [cited 2021 Dec 2]. Available from: https://apps.who.int/iris/bitstream/
handle/10665/204475/WHO_ZIKV_MOC_16.3_por.pdf; jsessionid=278428E2C79E328FF776086F5A6E010A? sequence $=8$

15. Nunes ML, Carlini CR, Marinowic D, Neto FK, Fiori HH, Scotta MC, et al. Microcephaly and Zika virus: a clinical and epidemiological analysis of the current outbreak in Brazil. J Pediatr (Rio J). 2016;92:230-40.

16. Brasil. Ministério da Saúde. Governo declara fim de emergência nacional pelo vírus Zika. [cited $2021 \mathrm{Dec} 2$ ]. Available from: https://agenciabrasil.ebc.com.br/geral/noticia/2017-05/ governo-declara-fim-de-emergencia-nacional-pelo-virus-zika

17. Brasil. Ministério da Saúde. Secretaria de Vigilância em Saúde. Síndrome congênita associada à infecção pelo vírus Zika: situação epidemiológica, ações desenvolvidas e desafios, 2015 a 2019. Bol Epidemiol. 2019;50 N Esp:1-31. [cited 2021 Dec 2]. Available from: http://plataforma.saude.gov.br/anomaliascongenitas/boletim-epidemiologico-SVS-especial-2019.pdf

18. Landis JR, Koch GG. The measurement of observer agreement for categorical data. Biometrics. 1977;33:159-74.

19. Oliveira WK, Cortez-Escalante J, Oliveira WT, Carmo GM, Henriques CM, Coelho GE, et al. Increase in reported prevalence of microcephaly in infants born to women living in areas with confirmed Zika virus transmission during the first trimester of pregnancy: Brazil, 2015. MMWR Morb Mortal Wkly Rep. 2016;65:242-7.

20. Magalhães-Barbosa MC, Prata-Barbosa A, Robaina JR, Raymundo CE, Lima-Setta F, Cunha AJ. Trends of the microcephaly and Zika virus outbreak in Brazil, January-July 2016. Travel Med Infect Dis. 2016;14:458-63.

21. Vargas A, Saad E, Dimech GS, Santos RH, Sivini MA, Albuquerque LC, et al. Characteristics of the first cases of microcephaly possibly related to Zika virus reported in the Metropolitan Region of Recife, Pernambuco State, Brazil. Epidemiol Serv Saude. 2016;25:691-700.

22. Cabral CM, Nóbrega ME, Leite PL, Souza MS, Teixeira DC, Cavalcante TF, et al. Clinical-epidemiological description of live births with microcephaly in the state of Sergipe, Brazil, 2015. Epidemiol Serv Saude. 2017;26:245-54.

23. Martins RS, Fróes MH, Saad LD, Ignácio Junior SM, Prado WD, Figueiredo EM, et al. Descriptive report of cases of congenital syndrome associated with Zika virus infection in the state of São Paulo, Brazil, from 2015 to 2017. Epidemiol Serv Saude. 2018;27:e2017382.

24. Almeida IM, Ramos CV, Rodrigues DC, Sousa AC, Nascimento ML, Silva MV, et al. Clinical and epidemiological aspects of microcephaly in the state of Piauí, northeastern Brazil, 20152016. J Pediatr (Rio J). 2019;95:466-74.

25. Ribeiro IG, Andrade MR, Silva JM, Silva ZM, Costa MA, Vieira MA, et al. Microcephaly in Piauí, Brazil: descriptive study during the Zika virus epidemic, 2015-2016. Epidemiol Serv Saude. 2018;27:e20163692. 
26. Araújo TV, Ximenes RA, Miranda-Filho DB, Souza WV, Montarroyos UR, Melo AP, et al. Association between microcephaly, Zika virus infection, and other risk factors in Brazil: final report of a case-control study. Lancet Infect Dis. 2018;18:328-36.

27. Rocha SG, Correia LL, Cunha AJ, Rocha HA, Leite AJ, Campos JS, et al. Zika virus infection and microcephaly: a case-control study in Brazil. Ann Glob Health. 2019;85:116.

28. Herber S, Silva AA, Sanseverino MT, Friedrich L, Ranieri TM, Favreto C, et al. Prevalence and causes of congenital microcephaly in the absence of a Zika virus outbreak in southern Brazil. J Pediatr (Rio J). 2019;95:600-6.

29. Brasil. Ministério da Saúde. Secretaria de Vigilância em Saúde. Sífilis 2020. Bol Epidemiol. 2020; N Esp:1-42. [cited 2021 Dec 2]. Available from: https://www.gov.br/saude/pt-br/assuntos/ media/pdf/2020/outubro/29/BoletimSfilis2020especial.pdf
30. Brasil. Ministério da Saúde. Secretaria de Vigilância em Saúde. Monitoramento dos casos de arboviroses urbanas transmitidas pelo Aedes Aegypti (dengue, chikungunya e zika), semanas epidemiológicas 1 a 26, 2020. Bol Epidemiol. 2020;51:145. [cited 2021 Dec 2]. Available from: http://plataforma. saude.gov.br/anomalias-congenitas/boletim-epidemiologicoSVS-28-2020.pdf

31. Brasil. Ministério da Saúde. Secretaria de Vigilância em Saúde. Departamento de Vigilância das Doenças Transmissíveis. Protocolo de vigilância e resposta à ocorrência de microcefalia e/ou alterações do sistema nervoso central (SNC). Brasília: Ministério da Saúde; 2016. [cited 2021 Dec 2]. Available from: https://www.ribeiraopreto.sp.gov.br/files/ssaude/pdf/ zika-cartilha-protocolo-microcefalia.pdf 